\title{
A comparative study of three validities computation methods for multimodel approach
}

\author{
Samia Talmoudi Ben Aoun ${ }^{1}$, Abdennacer Ben Messaoud ${ }^{2}$, Moufida Lahmari Ksouri ${ }^{3}$ \\ ${ }^{1}$ Higher Institute of Applied Sciences and Technology of Mateur, University of Carthage, Tunisia \\ ${ }^{2,3}$ National Engineering School of Tunis, University of Tunis El Manar, Tunisia
}

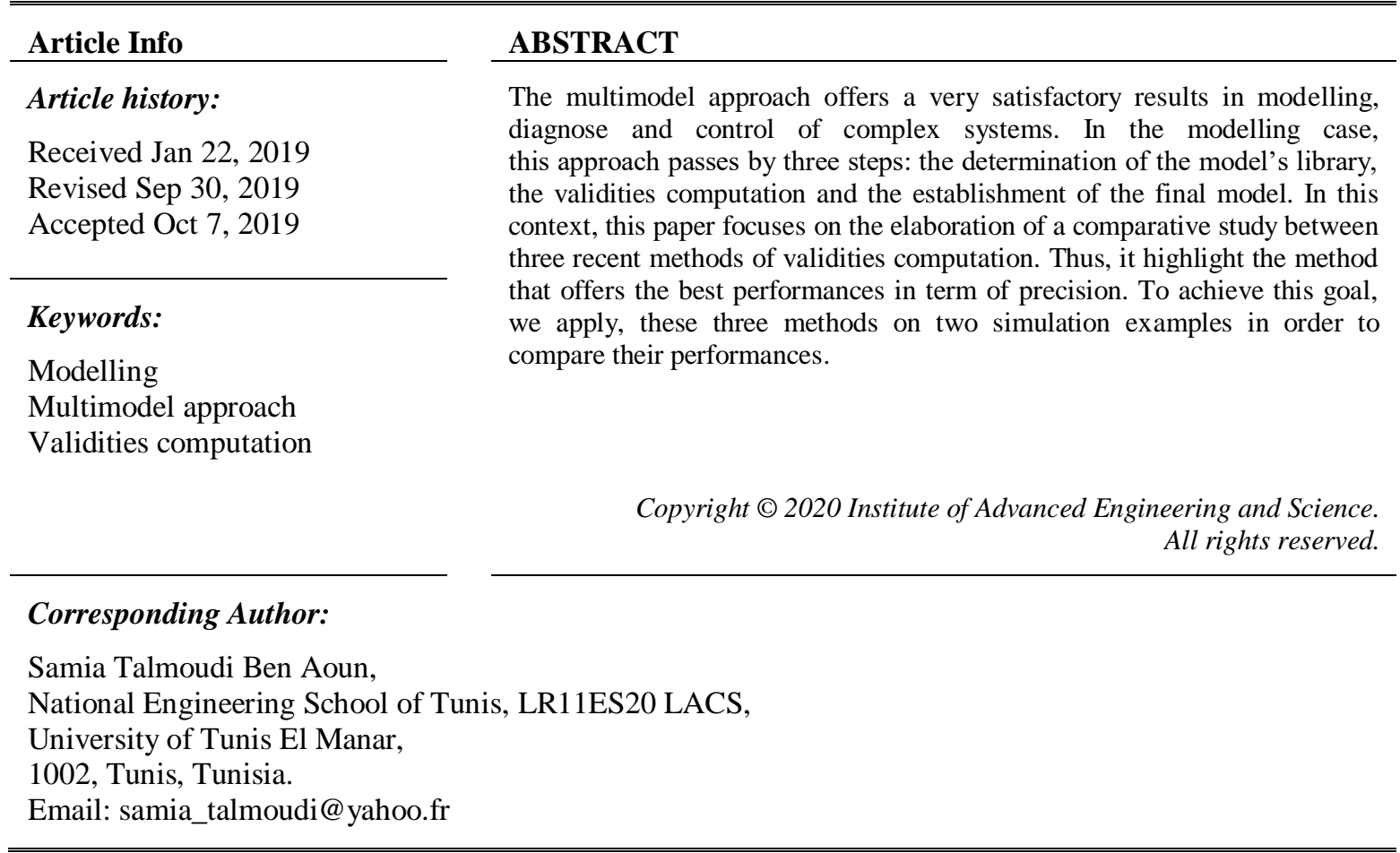

\section{INTRODUCTION}

The establishment of a mathematical model is the first concerns of researchers for application of the advanced techniques of analyses, monitoring, prediction, control and diagnose of complex systems [1, 2]. The multimodel approach has proved a very satisfactory results and a potential benefit in both modelling and identification of complex, nonlinear and/or ill-defined systems, compared to a "single model approach". Indeed, the "single model approach" consists in determining one model that describes the comportment of the system in all its operating regions. This mission is very difficult and can sometimes be impossible when the system includes set-point changes or/and the co-existence of multiple operating modes [3, 4]. Although, the multimodel approach consists on partitioning the global system's full range operation into multiple smaller ranges. To each range is associated a local model that describes the system behavior in this specific range. The set of the local models forms the called models-library or models-base. A coefficient called validity is associated to each local model of the models-library. Validity estimates each library-model contribution in the reproduction of the real process behavior. Several validities' computation methods have been proposed in the literature [5-13]. These methods depend firstly on the way the library-models were determined and secondly, on the information available on the system. We distinguish two major classes of validities. Firstly the a priori validities which can be determined offline by exploiting the a priori knowledge available on the system. Secondly, the a posteriori validities which must be calculated online by considering the measures carried out at each instant. The present paper is interested by a postoriori validity. The majority of methods belonging to the last class are based on the residues computation and are established by measuring omline, at each instant, the distance between the process output and those of the variaous models of the base. These methods are suitable when there is overlapping between data of the different models of 
the the models'base [14]. Besides, these methods are limited and cannot be useful with presence of complex and/or ill-defined system. To overcome these problems, Ben Messaoud and all. Have proposed recently, three new methods of computation validities. In fact, the first method is based on a local criterion optimization LCO [15]. The second method uses a hierarchical structuring HS to compute validities [16]. In the third method, validities based on optimal computation OC, are obtained by optimizing a constrained least squares problem [6]. In the present paper, we focus our study to compare the last three methods and to determine the one leads to the best model that can describe perferctly the real process. In order to compare performances assumed by the three proposed methods of validities computation, we consider two numerical simulation examples. In the second part of the paper, we present the general structure of the multimodal approach. The third section discusses the three considered calculation validities methods. Two examples of numerical simulation are given in section four. We finish this work by a conclusion.

\section{GENERAL STRUCTURE OF MULTIMODEL APPROACH}

The general structure of the multimodel approach is presented in Figure 1. It is formed by three units: the models library unit, the decision unit and the output unit [12, 17, 18].

\subsection{Models library unit}

This unit can contain different types of models (either input-output models or state space models). These models are distinguished with respect to four features: Partition strategy, submodel structural identification, transition between models and method of realization [19-21].

\subsection{Decision unit}

This unit is responsible of computing models validities $v_{i}$. It is a fundamental unit as it determines the degrees of contribution of each local model of the library in the composition of the output of the original system. It is assumed that the contribution of all local models' sum is equal to 1 anywhere across the operating space $[11,21]$.

$$
\begin{aligned}
& v_{i} \in[0,1] ; i=1,2, \ldots, L \\
& \sum_{i=1}^{L} v_{i}=1
\end{aligned}
$$

Where $L$ represent the number of library' models

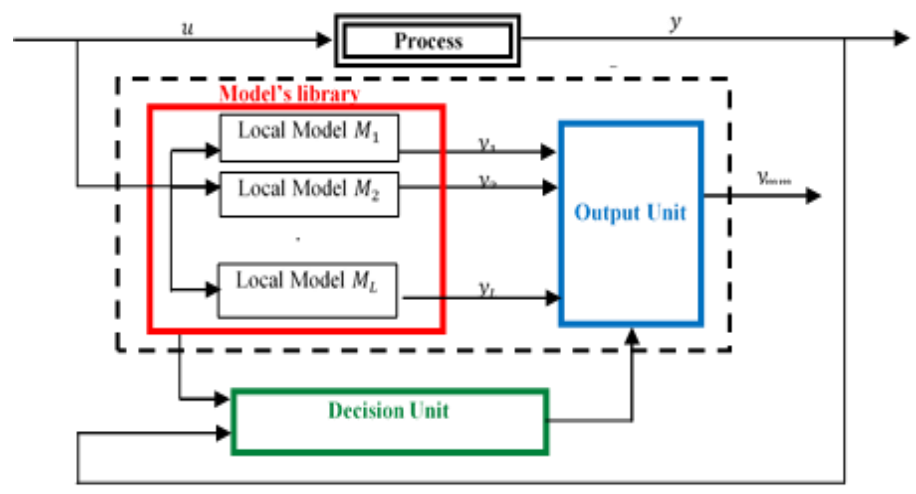

Figure 1. General structure of system modelling by multimodel approach

\subsection{Output unit}

The output unit computes the multimodel output $y_{m m}$ by exploiting outputs obtained by modelslibrary unit and the decision unit as follows:

$$
y_{m m}(k)=\sum_{i=1}^{L} v_{i}(k) y_{i}(k)
$$

Where $\mathrm{y}_{\mathrm{i}}$ is the output of the model $M_{i}$ of the models-library and $L$ is the number of library' models. 


\section{VALIDITIES COMPUTATION}

\subsection{The first Method: Validities computation by local criterion optimization LCO}

This method is presented by Ben Messaoud and al in [15]. In this method, the validity $v_{i}$ can be estimated, at each instant by minimizing the criterion given by the (4), which represent an equality constrained optimization problem [16]:

$$
\begin{gathered}
J=\frac{1}{2} \sum_{i=1}^{L} v_{i}{ }^{2}(k)\left(y(k)-y_{i}(k)\right)^{2} \\
\text { subject to: } \sum_{j=1}^{L} v_{j}(k)=1
\end{gathered}
$$

Where: $L$ is the number of library' models.

$y_{i}(k)$ is the output of $i^{\text {th }}$ local model,

$y(k)$ is the measured output of the nonlinear system.

The solution of the optimization criterion above is given by the following expression:

$$
v_{i}(k)=\frac{\frac{1}{r_{i}^{2}(k)}}{\sum_{j=\frac{1}{r_{j}}{ }^{2}(k)}^{\frac{1}{2}}} ; i=1, \ldots, L
$$

Where $r_{i}(k)$ represents the residue at the instant $k$ and is based on the online computation of the difference between the process output and those of the various models $M_{i}$ of the base at the instant $k$ as below:

$$
r_{i}=\left|y-y_{i}\right| ; i=1, \ldots, L
$$

\subsection{The Second Method: Validities computation based on an hirarchic structure HS}

This method is described by Ben Messaoud and al. in [16] and is summarized as follows:

Let us assume that at instant $k$, the residues' calculation using the (6) gives ascending values $\left(r_{1} \leq r_{2} \leq \cdots \leq r_{L}\right)$. The second method for validities computation is based on a quasi-hierarchical structuring whose validities are calculated by the following equation:

$$
v_{i}^{\operatorname{simp}}=\frac{v_{i}}{\sum_{j=1}^{L} v_{j}} ; i=1, \ldots, L
$$

Where:

$$
v_{i}=1-\frac{r_{i}}{\sum_{j=1}^{L} r_{j}} ; i=1, \ldots, L
$$

In the general case and for each instant $\mathrm{k}$, the base-models' validities are computed by using the following algorithm:

Step 1: Create a table containing all base-models' outputs:

$$
T a b_{y}=\left[\begin{array}{llll}
y_{1} & y_{2} & \ldots & y_{L}
\end{array}\right]
$$

Step 2: Calculate the corresponding residues by (6).

Step 3: Create a table containing all the obtained residues:

$$
T a b_{r}=\left[\begin{array}{llll}
r_{1} & r_{2} & \ldots & r_{L}
\end{array}\right]
$$

Step 4: Arrange $T a b_{r}$ in ascending order into $T a b C_{r}$ and create a table $T a b_{i n d}$ for the corresponding indices Step 5: of $T a b_{r}$

$$
\begin{aligned}
& \text { for } \mathrm{i}=1 \text { to } 2 \\
& \quad r_{i}^{\prime} \leftarrow \operatorname{Tab} C_{r}[i] \\
& \quad \text { Calculate } v_{i}^{\prime} \text { by (7) } \\
& \text { End }
\end{aligned}
$$


$y_{p m m_{1}}=v_{1}^{\prime} \cdot T a b_{y}\left[\operatorname{Tab}_{\text {ind }}[1]\right]+v_{2}^{\prime} \cdot \operatorname{Tab}_{y}\left[\operatorname{Tab}_{\text {ind }}[2]\right]$

$$
j \leftarrow 1
$$

for $\mathrm{i}=3$ to $\mathrm{L}$

$r_{i}^{\prime} \leftarrow T a b C_{r}[i] ; \quad r_{p m m_{j}}=\left|y-y_{p m m_{j}}\right|$

Calculate $v_{i}^{\prime}$ and $v_{p m m_{j}}$ by (7)

if $\mathrm{i}==\mathrm{L}$

STOP!

else

$y_{p m m_{j+1}}=v_{p m m_{j}} \cdot y_{p m m_{j}}+v_{i}^{\prime} \cdot \operatorname{Tab}_{y}\left[\operatorname{Tab}_{\text {ind }}[i]\right]$

end

$j \leftarrow j+1$

end

Step 6: Calculate the validities of the different models from the following loop:

for $\mathrm{i}=1$ to $\mathrm{L}$

for $\mathrm{j}=1$ to $\mathrm{L}$

if $T a b_{i n d}[j]==\mathrm{i}$

if $j==1$

$v_{i}=v_{j}^{\prime}\left(\prod_{k=1}^{L-2} v_{p m m_{k}}\right)$

else if $j==L$

$v_{i}=v_{L}^{\prime}$

else

$v_{i}=v_{j}^{\prime}\left(\prod_{k=j-1}^{L-2} v_{p m m_{k}}\right)$

\section{End if \\ End if \\ End for \\ End for}

\subsection{The Third Method: validities' optimal computation OC}

The third proposed method of validities computation $v_{i}$ is based on the optimization of a constrained least squares problem [6]. The formulation of the quadratic programming is as follows:

$$
\begin{aligned}
& \min _{\mathrm{v}} \frac{1}{2} \mathrm{v}^{T} \mathrm{Gv}+\mathrm{c}^{T} \mathrm{v} \\
& \text { subject to: }\left\{\begin{array}{l}
\mathrm{Dv}=h \\
\mathrm{Fv} \geq \mathrm{e}
\end{array}\right.
\end{aligned}
$$

Where: $\mathrm{D}=\left(\begin{array}{llll}1 & 1 & \cdots & 1\end{array}\right) \in 1 \times L, h=1, \mathrm{~F}=\left(\begin{array}{c}-\mathrm{I}_{L \times L} \\ \mathrm{I}_{L \times L}\end{array}\right)$ and $\mathrm{e}=\left(\begin{array}{c}-1 \\ \vdots \\ -1 \\ 0 \\ \vdots \\ 0\end{array}\right) \in 2 L \times 1$;

$G=A^{T} A$ and $c=-A^{T} b$.

The active-set method is chosen for solving the problem above [17]. The details of validities' computation by this method are given by the following algorithm:

Step 1 Determine an initial feasible point $v^{0}$ by solving the linear programming problem below (16), and by using the simplex method: 
$\min _{(v, z)} w^{T} z$
subject to: $\left\{\begin{array}{c}d_{i}^{T} v+\gamma_{i} z_{i}=h_{i} ; i=1 \\ f_{j}^{T} v+\gamma_{j+1} z_{j+1} \geq e_{j} ; j=1, \ldots, 2 L \\ z \geq 0\end{array}\right.$

where: $w=\left(\begin{array}{llll}1 & 1 & \cdots & 1\end{array}\right)^{T} \in(2 L+1) \times 1$,

$$
\gamma_{i}=-\operatorname{sign}\left(d_{i}{ }^{T} \tilde{v}-h_{i}\right) \text { for } i=1
$$

and $\gamma_{j+1}=1$ for $j=1, \ldots, 2 L$ with $\tilde{v}$ is an initial estimate of the vector $v$.

Step 2 Determine the working set $W^{0}$ which contains the indexes representing the equality and active inequality constraints:

$$
W^{0}=\left\{i \mid \mathrm{d}_{i}^{T} \mathrm{v}^{0}=h_{i}\right\} \cup\left\{j \mid \mathrm{f}_{j}^{T} \mathrm{v}^{0}=e_{j}\right\}
$$

for $\quad l=0,1,2, \ldots$ do

Find the search direction $p^{l}$ and the Lagrange multipliers $\lambda^{1}$ and $\mu^{l}$ by solving KKT-equations (Karush-Kuhn-Tucker) of the quadratic subproblem:

$$
\begin{aligned}
& \min _{p} \frac{1}{2} p^{T} G p+\left(G v^{l}-c\right)^{T} p \\
& \text { subject to: } \\
& \begin{array}{l}
d_{i}^{T} p=0 ; i \in W^{l} \\
f_{j}^{T} p=0 ; j \in W^{l}
\end{array} \\
& \text { if } \quad p^{l}=0 \text { then } \\
& \text { if } \quad \begin{array}{l}
\mu^{l} \geq 0 \text { then } \\
\text { STOP! With } v^{*}=v^{l} \text { is the optimal solution; }
\end{array} \\
& \text { else } \quad
\end{aligned}
$$

Find the most negative component of $\mu^{l}$ :

$$
\begin{aligned}
& \mu_{j_{0}}^{l}=\min \left\{\mu_{j}^{l} \mid \mu_{j}^{l}<0, j \in W^{l}\right\} \\
& v^{l+1}=v^{l} ;
\end{aligned}
$$

Remove constraint corresponding to the most negative component of $\mu^{l}$ from end if the working set: $W^{l+1} \leftarrow W^{l} \backslash\left\{j_{0}\right\}$;

else $\left\{p^{l} \neq 0\right\}$

Compute the step-length $\alpha^{l}$ that guarantees the satisfaction of all constraints from:

$\alpha^{l}=\min \left(1, \frac{e_{j}-\mathrm{f}_{j}^{T} \mathrm{v}^{l}}{\mathrm{f}_{j}^{T} \mathrm{p}^{l}} \mid j \notin W^{l}\right.$ and $\left.\mathrm{f}_{j}^{T} \mathrm{p}^{l}<0\right)$

Update: $v^{l+1} \leftarrow v^{l}+\alpha^{l} p^{l}$;

Update $W^{l}$ :

$$
\text { if } \alpha^{l}=1 \text { then }
$$

$$
W^{l+1} \leftarrow W^{l} ;
$$

else

Add one constraint to the working set: $\mathrm{W}^{1+1} \leftarrow \mathrm{W}^{1} \cup\left\{\mathrm{j}_{0}\right\}$

where

$$
\alpha^{l}=\frac{e_{j_{0}}-\mathrm{f}_{j_{0}}{ }^{T} \mathrm{v}^{l}}{\mathrm{f}_{j_{0}}{ }^{T} \mathrm{p}^{l}} \text { for } \mathrm{f}_{j_{0}}{ }^{T} \mathrm{p}^{l}<0 ;
$$

end if

$$
\text { end if }
$$

end for 


\section{SIMULATION EXEMPLES}

To compare the performances offered by the three considered computation validities methods, we propose two numical simulation examples.

\subsection{Exemple 1}

This first example is a discrete system with time varying parameters $a_{1}(k), a_{2}(k), b_{1}(k)$ and $b_{2}(k)$ described by the $(21)[11,22,23]$. The variation laws of the process parameters are given by Figure 2 .

$$
y(k)=-a_{1}(k) y(k-1)-a_{2}(k) y(k-2)+b_{1}(k) u(k-1)+b_{2}(k) u(k-2)
$$

By applying the multimodel approach, Talmoudi et al. [18] has established the following models-library transfer functions:

$$
\begin{aligned}
& H_{1}\left(z^{-1}\right)=\frac{0.18104 z^{-1}+0.071832 z^{-2}}{1-1.1657 z^{-1}+0.2073 z^{-2}} \\
& H_{2}\left(z^{-1}\right)=\frac{0.10423 z^{-1}+0.1325 z^{-2}}{1-1.2806 z^{-1}+0.3258 z^{-2}} \\
& H_{3}\left(z^{-1}\right)=\frac{0.018301 z^{-1}+0.20512 z^{-2}}{1-1.3801 z^{-1}+0.42936 z^{-2}}
\end{aligned}
$$
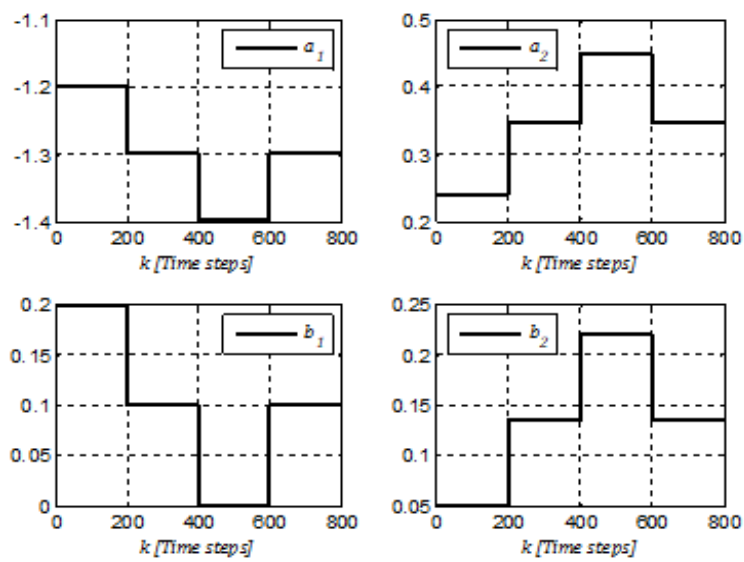

Figure 2. Evolution of parametres variation laws

After determining the library-models, we compute the validities degrees of each model of the library by using the three methods described above. The evolutions of validities degrees obtained by using LCO, HS and OC methods are presented, respectively, in Figures 3, 4 and 5. These Figures show that OC method gives the best results in term of precision. Indeed, it is clear, from the considered system (20), that at each range of time, there is only one transfer function that is totally valid. Or, Only the OC method demonstrates this feature in Figure 5. LCO and HS methods give for each range of time one model having a validity degree near to 1 but not equal to 1 .

The multimodel output is, then, obtained by the fusion of the different models outputs $y_{1}(k), \mathrm{y}_{2}(\mathrm{k})$ and $y_{3}(k)$ weighted by their respective validity degrees computed by the different proposed methods:

$$
\begin{aligned}
& y_{m m L C O}(k)=v_{1 \mathrm{LCO}}(k) y_{1}(k)+v_{2 \mathrm{LCO}}(k) y_{2}(k)+v_{3 \mathrm{LCO}}(k) y_{3}(k) \\
& y_{m m H S}(k)=v_{1 H S}(k) y_{1}(k)+v_{2 H S}(k) y_{2}(k)+v_{3 H S}(k) y_{3}(k) \\
& y_{m m o C}(k)=v_{1 O C}(k) y_{1}(k)+v_{2 O C}(k) y_{2}(k)+v_{3 O C}(k) y_{3}(k)
\end{aligned}
$$

Where:

- $y_{m m L C O}, y_{m m H S}, y_{m m o c}$ are multimodel ouputs using respectively LCO, HS and OC methods.

- $\quad \mathrm{v}_{1 \mathrm{LCO}}(\mathrm{k}), \mathrm{v}_{2 \mathrm{LCO}}(\mathrm{k})$ and $\mathrm{v}_{3 \mathrm{LCO}}(\mathrm{k})$ are validities for LCO method.

- $\quad \mathrm{v}_{1 \mathrm{HS}}(\mathrm{k}), \mathrm{v}_{2 \mathrm{HS}}(\mathrm{k})$ and $\mathrm{v}_{3 \mathrm{HS}}(\mathrm{k})$ are validities for HS method.

- $\quad \mathrm{v}_{10 \mathrm{CC}}(\mathrm{k}), \mathrm{v}_{20 \mathrm{C}}(\mathrm{k})$ and $\mathrm{v}_{30 \mathrm{C}}(\mathrm{k})$ are validities for OC method. 
Figure 6 shows the evolutions of the relative errors obtained when we apply the three considered validities computation methods. It is clear that OC method offers the best precision by comparison to the two other methods.

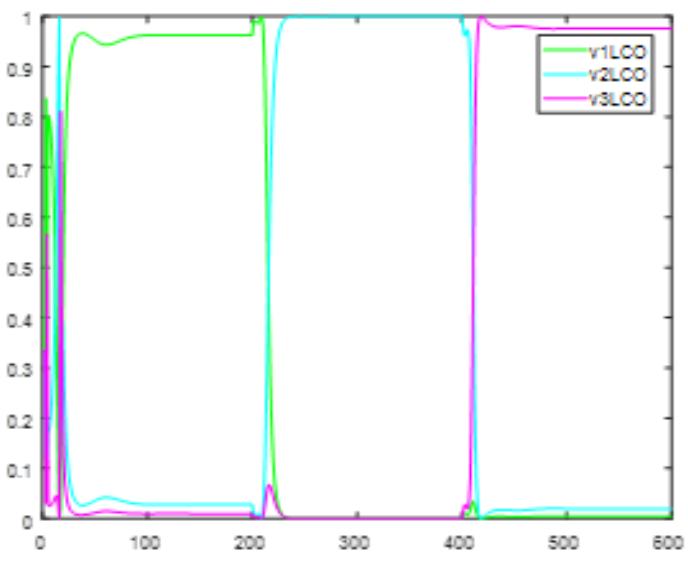

Figure 3. Validities evolution of LCO method

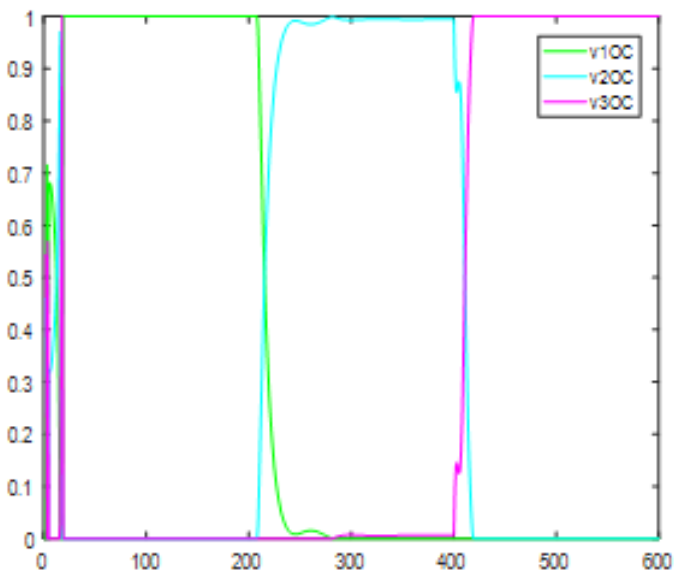

Figure 5. Validities evolution of OC method

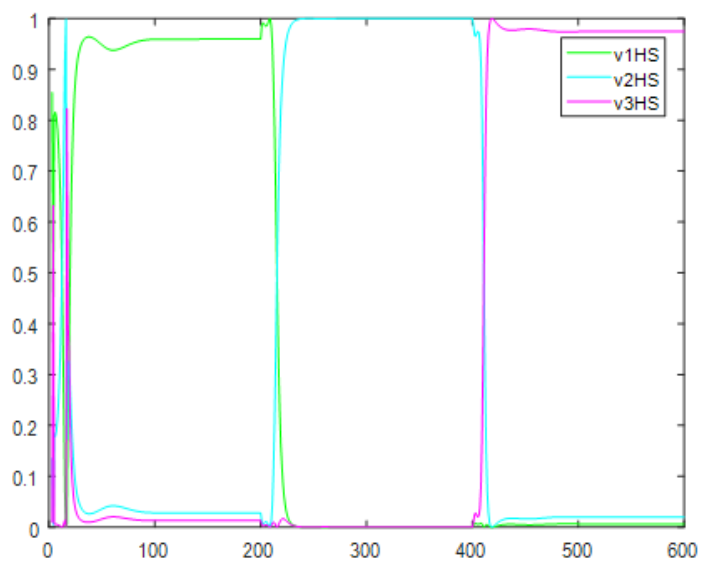

Figure 4. Validities evolution of HS method

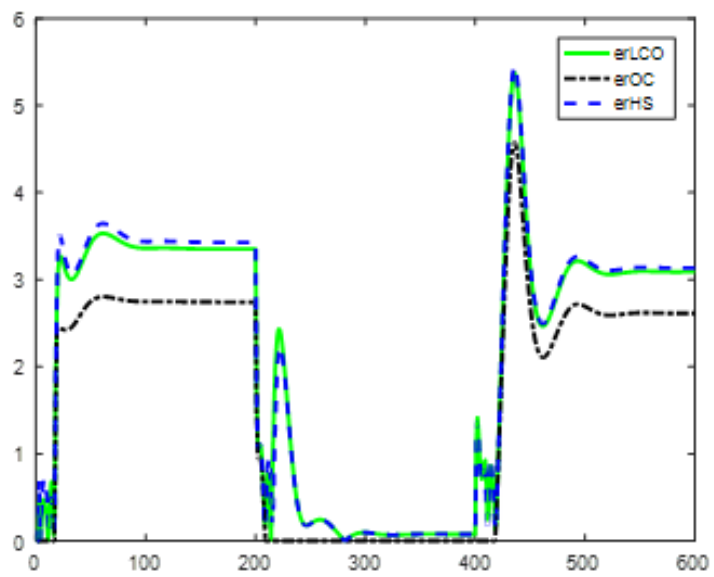

Figure 6. Relative errors evolutions

\subsection{Exemple 2}

The second considered example is an uncertain complex system represented by the following equation $[6,20,18]$ :

$$
x(t)+\tau(x) \dot{x}(t)=k(x) u(t) ; x \in[0,1]
$$

Where: $k(x)=36 x(t)(x(t)-1)+10$ and $\tau(x)=15-10 x(t)$.

It is clear that the considered system is nonlinear. Indeed, the gain and the time constant vary with $x$. This nonlinearity appears more in Figures 7 and 8. The last Figures shows the evolution of the time constant and the gain variations.

The minimal and maximal values of respectively the gain and the time constant are:

$$
k_{\min }=1 ; k_{\max }=10 ; \tau_{\min }=5 ; \tau_{\max }=15 ;
$$

By using the Kharitonov's algebraic approach [9], we obtain the following models' library: 


$$
\begin{array}{ll}
F_{1}(s)=\frac{1}{1+15 s} ; & F_{2}(s)=\frac{1}{1+5 s} \\
F_{3}(s)=\frac{10}{1+15 s} ; & F_{4}(s)=\frac{10}{1+5 s}
\end{array}
$$

The system (27) and the four transfer functions (29) are implanted in the Matlab Simulink environment. Matlab discretize these equations by using the Bogacki-Shampine method with sampling time $T_{s}$ equal to $0.1 \mathrm{~s}$.

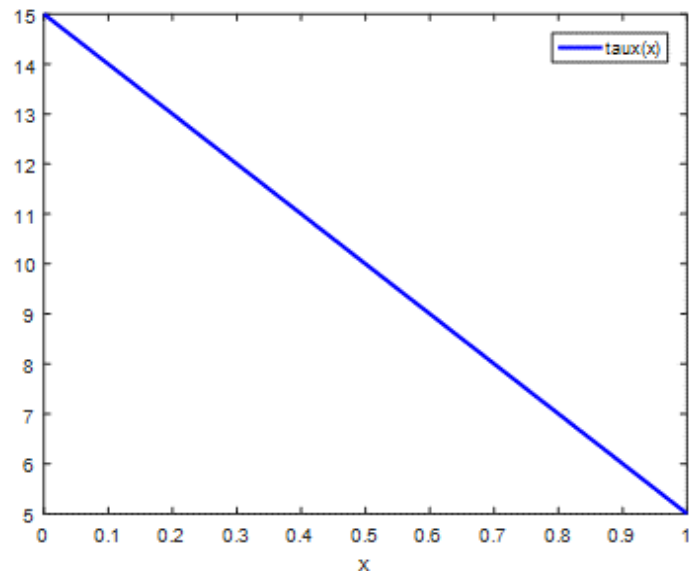

Figure 7. The constant time variation

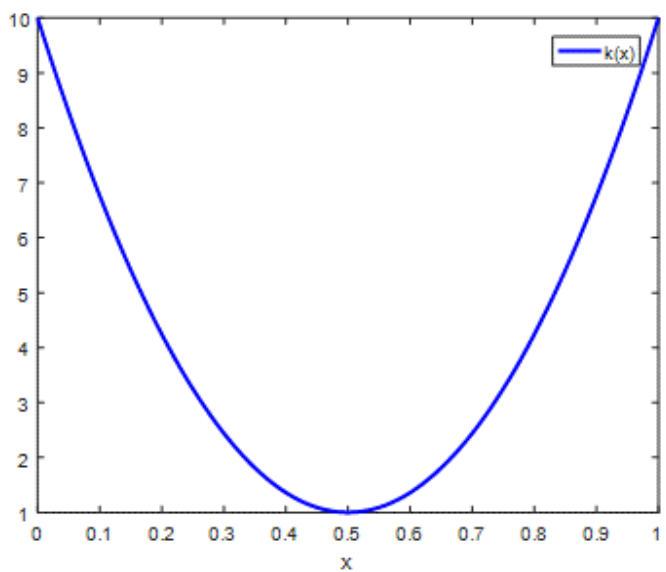

Figure 8. The gain variation

The Figure 9 shows the evolutions of the process and the multimodel states obtained by using the three computation validities methods and by applying a unit step at the input. It is clear the multimodel state for OC method coincides perfectly with the process state. Although the two other states relative to LCO and HS methods follows the process state but with an error relatively important. These errors are represented in Figure 10 which shows that the relative error correspondent to OC method is equal to 0 at every instant. The other relative errors are relatively important.

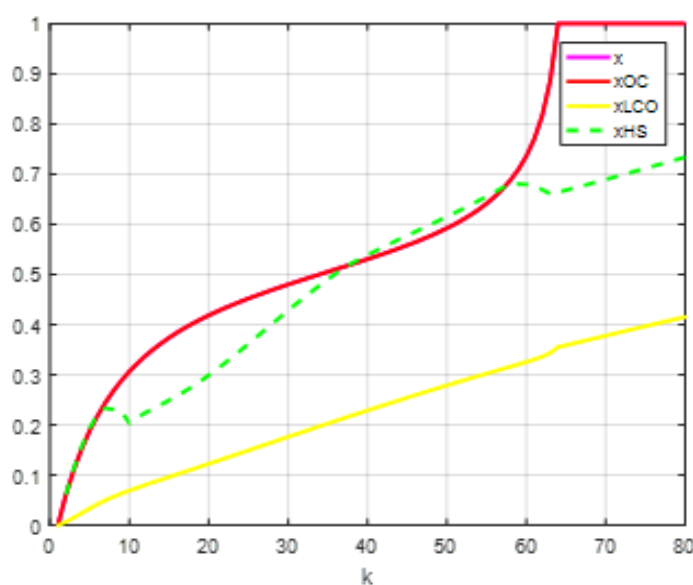

Figure 9. Process and multimodel states

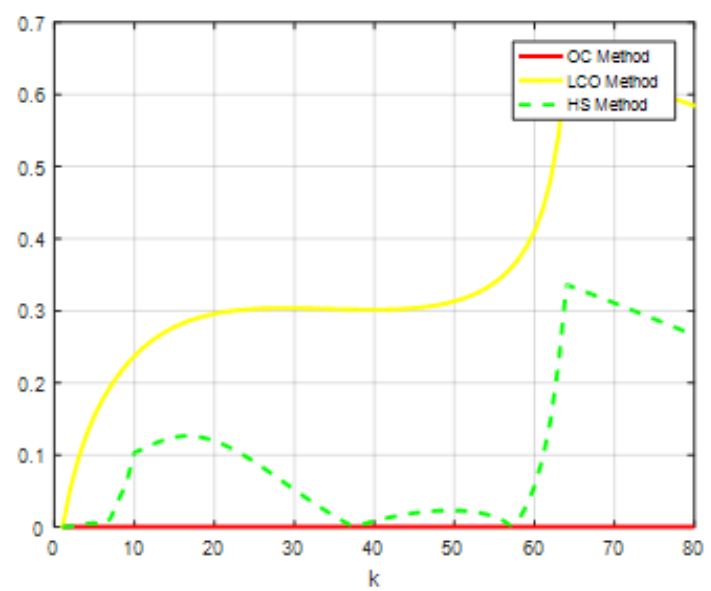

Figure 10. Relative errors evolutions

Where: $x$ represents the process state

$x_{L C O}$ is the multimodel state obtained by using LCO method,

$x_{H S}$ is the multimodel state obtained by using HS method,

$x_{O C}$ is the multimodel state obtained by using OC method. 


\section{CONCLUSION}

On our research, we are interested by exploiting the multimodel approach for modelling complex, non-linear or/and uncertain systems. This paper presents a large comparative study of three validities computation methods. These methods are recently proposed by Ben Messaoud and al. We are succeeded to prove that the method based on resolving an optimization problem by using the active set method i.e OC method, gives the best results of modelling with a good precision.

\section{REFERENCES}

[1] Marwa Hannachi, Ikbel Bencheikh Ahmed, and Dhaou Soudani, "Discrete-time Inversion Model Control of a Double-damper System with Uncertain Parameters," International Journal of Robotics and Automation (IJRA), vol. 6(3), pp. 168-177, Sep 2017.

[2] Dana Dehghani, Azli Yahya, Nor Hisham Khamis, and Ali Idham Alzaidi, "EDM Process through Mathematical Model," International Journal of Power Electronics and Drive System (IJPEDS), vol. 10(2), pp. 874-881, 2019.

[3] Ahmed Saïd Nouri, Mohamed Mihoub, and Ridha Ben Abdennour, "Multimodel discrete sliding mode control for non-stationary systems," Int. J. Modelling, Identification and Control, vol. 3(4), pp. 368-375, 2008.

[4] Ahmed Rhif, Zohra Kardous, and Naceur BenHadj Braiek, "A sliding mode multimodel control for a sensorless photovoltaic system," Journal of Scientific \& Industrial Research, vol. 71, pp. 418-424, 2012.

[5] Abdallah Salem, Ali Sghaïer Tlili and Naceur Benhadj Braiek, "On the plotytopic and multimodel state observers of inductor motor," Journal of Automation \& Systems Engineering, vol. 2, no. 4, 2008.

[6] Ben Messaoud Abdennacer, Talmoudi Samia, and Moufida Ksouri., "Multimodel approach for modelling of nonlinear systems: validities' optimal computation," International journal for Computation and Mathematics in Electrical and Electronic Engineering, vol. 37(1), pp. 153-175.

[7] Chadli M., "Stability and control of systems described by multimodel (in French)," Thèse de doctorat, Institut National Polytechnique de Lorraine, 2002.

[8] H. Gharsallaoui, "Reconfiguration of control laws and active accommodation of operating modes for flat systems (in French)," Thèse de doctorat, Ecole centrale de Lille en cotutelle avec l'école nationale d'ingénieurs de Tunis, 2010.

[9] Nawel Mensia and Moufida Ksouri, "Adaptive Neural Internal Model Control for Complex Process with Delay, International Conference on Communications," Computing and Control Applications (CCCA), pp. 1-7, 2011.

[10] R. Orjuela, B. Marx, J. Ragot, and D. Maquin, "State estimation of nonlinear systems based on heterogeneous multiple models: some recent theoretical results," 3rd IFAC Workshop on Advanced Fuzzy and Neural Control, vol. 3(1), pp. 97-102, 2007.

[11] Talmoudi S, Ben abdennour R, Abderrahim K, and Borne P., "A Systematic Determination Approach of a Models' Base for Uncertain Systems: Experimental Validation," Systems, Man and Cybernetics, vol. 6, 2002.

[12] Verdult V, Ljung L, and Verhaegen M., "Identification of composite local linear state-space models using a projected gradient search," International Journal of Control, vol. 75(16/17), pp. 1385-1398, 2002.

[13] Saïda Bedoui, Majda Ltaief, and Kamel Abderrahim., "New method for the systematic determination of the model's base of time varying delay system," International Journal of Computer Applications, vol. 46(1), pp. 13-19, 2012

[14] Elfelly, N, Dieulot, J.Y, and Borne P., "A neural approach of multimodel representation of complex processes," International Journal ofComputers Communications\&Control, vol. 3(2), pp. 149-160, 2008.

[15] Ben Messaoud Abdennacer, Talmoudi Samia, and Moufida Ksouri., "Multimodel Approach: Validities computation by local criterion optimization," International conference of advanced systems and electric Technologies 14-17 January 2017, Hammamet, Tunisia.

[16] Ben Messaoud Abdennacer, Talmoudi Samia, and Moufida Ksouri., "A new strategy of validities computation for multimodel approach: Experimental validation," International Journal of Advanced Computer Science and Applications (IJACSA), vol. 8(7), Jul 2017.

[17] Shweta Goyal, Sachin Mishra, and Anamika Bhatia, "A comparative approach between different optimize result in hybrid energy system using HOMER," International Journal of Electrical and Computer Engineering (IJECE), vol. 9(1), pp. 141-147, Feb. 2019.

[18] M. Ltaief, A. Messaoud, and R. Ben Abdennour, "Optimal systematic determination of models' base for multimodel representation: real time application," International Journal of Automation and Computing, vol. 11(6), pp. 644-652, Dec 2014.

[19] V. D. S. Martins, M. Rodrigues, and M. diagne, "A multi-model approach to Saint-Venant equations: A stability study by LMIs," International Journal of Applied Mathematics and Computer Science, vol. 22(3), pp. 539-550, 2012.

[20] Delmotte F, "Multi-model analysis (in French)," Thèse de doctorat, Université des sciences et techniques de Lille, 1997.

[21] S. El Feik and A. Adeniran, "Modeling and identification of nonlinear systems: a review of the multimodel approach-part 2," IEEE Transactions of systems, man, and cybernitics: systems, vol. 47(7), Jul 2017.

[22] R. Orjuela, B. Marx, J. Ragot, and D. Maquin, "Nonlinear system identification using heterogeneous multiple models," International Journal of Applied Mathematics and Computer Science, vol. 23(1), pp. 103-115, 2013. 
[23] Talmoudi S, Abderrahim K, Ben abdennour R, and Ksouri M., "Multimodel approach using neural networks for complex systems modeling and identification," Nonlinear Dynamics and Systems Theory, vol. 8(3), pp. 299-316, 2008.

\section{BIOGRAPHIES OF AUTHORS}

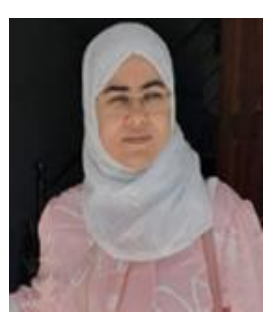

Samia Talmoudi has received her Ph.D. degree in electrical-automatic engineering from National School of Engineering of Tunis in 2005. From 2005 to 2015, she was an associate professor in the Electronic Department, at the Higher Institute of Sciences and Technology of Mateur, Tunisia. Actually, she is an associate professor in University of Tabuk, Arabia Saoudite. She is interested with multimodel and multicontrol approaches, neural networks, fractional systems modeling, and numerical control of complex systems.

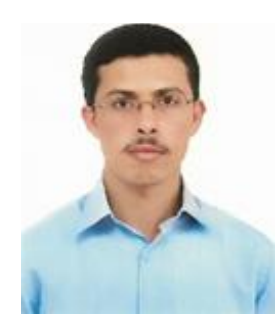

Abdennacer Ben Messaoud received his Engineering Diploma in Electric and Automatic Engineering, in 2002, and Master degree in Automatic Control and Intelligent Techniques, in 2009, from National School of Engineers of Gabes (ENIG), Tunisia. He has over 13 years of industrial experience. Currently, he is pursuing his $\mathrm{PhD}$ thesis at Laboratory of Analysis Conception and Control Systems (LACS) at National Engineering School of Tunis (ENIT), Tunisia. His research interests include multimodel approach and modelling of fractional-order systems.

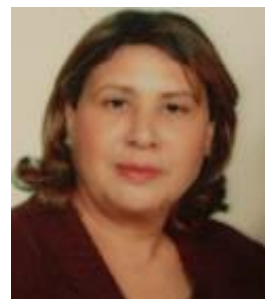

Moufida Ksouri is a professor at the National School of Engineering of Tunis. She received her Ph.D. degree in Electrical Engineering from the University of Science and Technology of Lille in 1998. Her research interests include Identification and control of non-linear systems and industrial applications of advanced control. She is the author or co-author of more than 30 papers in international conferences and journals. She has also published a book entitled "Commande numérique des processus". 